\title{
Verbal working memory modulates afferent circuits in motor cortex
}

\author{
Lorraine Y. Suzuki | Sean K. Meehan
}

Human Sensorimotor Laboratory, School of Kinesiology, University of Michigan, Ann Arbor, Michigan

\section{Correspondence}

Sean K. Meehan, Department of

Kinesiology, University of Waterloo,

Waterloo, ON.

Email: skmeehan@uwaterloo.ca

Present Address

Department of Kinesiology, University of

Waterloo, Waterloo, ON, Canada

Funding information

National Institute on Aging, Grant/Award

Number: AG024824

\begin{abstract}
Verbal instruction and strategies informed by declarative memory are key to performance and acquisition of skilled actions. We previously demonstrated that anatomically distinct sensory-motor inputs converging on the corticospinal neurons of motor cortex are differentially sensitive to visual attention load. However, how loading of working memory shapes afferent input to motor cortex is unknown. This study used short-latency afferent inhibition (SAI) to probe the effect of verbal working memory upon anatomically distinct afferent circuits converging on corticospinal neurons in the motor cortex. SAI was elicited by preceding a suprathreshold transcranial magnetic stimulus (TMS) with electrical stimulation of the median nerve at the wrist while participants mentally rehearsed a two- or six-digit numeric memory set. To isolate different afferent intracortical circuits in motor cortex SAI was elicited, using TMS involving posterior-anterior (PA) or anterior-posterior (AP) monophasic current. Both PA and AP SAI were significantly reduced during maintenance of the six-digit compared to two-digit memory set. The generalized effect of working memory across anatomically distinct circuits converging upon corticospinal neurons in motor cortex is in contrast to the specific sensitivity of AP SAI to increased attention load. The common response across the PA and AP SAI circuits to increased working memory load may reflect an indiscriminate perisomatic mechanism involved in the voluntary facilitation of desired and/or suppression of unwanted actions during action selection or response conflict.
\end{abstract}

\section{K E Y W O R D S}

anterior-posterior, posterior-anterior, short-latency afferent inhibition, somatosensory-evoked potential, transcranial magnetic stimulation

\section{1 | INTRODUCTION}

Acquisition and execution of a motor skill is dependent upon the afferent input to motor cortex. Afferent input can be shaped by volitional strategy through the allocation of cognitive resources. For example, the allocation of attention across space and/or sensory modality can enhance or decline motor performance (Meehan, Legon, \& Staines,

\footnotetext{
Abbreviations: $\mathrm{Ag}$ - $\mathrm{AgCl}$, silver-silver chloride; $\mathrm{AP}$, anterior-posterior; GABA, $\mathrm{\gamma}$-aminobutyric acid; MEP, Motor-evoked potential; PA, posterior-anterior; SAI, short-latency afferent inhibition; TMS, transcranial magnetic stimulation.

Edited by Gregor Thut and Reviewed by Aimee Nelsona and Tuomas Mutanen.

All peer review communications can be found with the online version of the article.
} 
2009), motor cortex excitability (Conte et al., 2007; Ruge, Muggleton, Hoad, Caronni, \& Rothwell, 2014) and potential for motor cortical plasticity (Kamke et al., 2012, 2014; Stefan, Wycislo, \& Classen, 2004). Short-latency afferent inhibition (SAI) (Tokimura et al., 2000) offers a method to probe the modulatory effects of somatosensory afference upon motor cortex excitability and plasticity. SAI involves preceding a transcranial magnetic stimulation (TMS) stimulus over motor cortex by electrical stimulation of the corresponding contralateral afferent peripheral nerve $(\sim 20 \mathrm{~ms}$ for distal muscles of the hand). The inhibition evoked by the electrical stimulation is thought to be cortical in origin and reflect the convergence of somatosensory afference and TMS-induced current upon the corticospinal neurons in motor cortex (Tokimura et al., 2000). The strong positive relationship between the magnitude of SAI and peripheral stimulus intensity (Bailey, Asmussen, \& Nelson, 2016; Fischer \& Orth, 2011) support the critical role of somatosensory afferent projections to motor cortex. In addition to SAI's strong relationship with the extrinsic properties of somatosensory afference, SAI is also modified by intrinsic processes. For example, SAI is greater in adjacent muscles not involved in an intended movement (Asmussen et al., 2014; Dubbioso, Raffin, Karabanov, Thielscher, \& Siebner, 2017; Voller et al., 2006). For muscles involved in a movement SAI decreases during movement planning and onset (Asmussen, Jacobs, Lee, Zapallow, \& Nelson, 2013; Asmussen et al., 2014; Voller et al., 2006). The apparent sensitivity of SAI to intrinsic processes makes SAI a potential method to probe the effect of cognition on motor cortex excitability through afferent modulation.

To date, the vast majority of studies have quantified SAI by pairing monophasic posterior-anterior (PA) TMS with the peripheral electrical stimulation. Anatomically, SAI evoked by PA and monophasic anterior-posterior (AP) current index different intracortical sensory-motor circuits (Ni et al., 2011). Functionally, we recently demonstrated that SAI evoked using $\mathrm{AP}$, but not PA, current is reduced by a concurrent visual detection task with high attention demands (Mirdamadi, Suzuki, $\&$ Meehan, 2017). The sensitivity of the AP sensitive circuit to cross-modal attention represents a motor cortical substrate by which cognition can exert influence over the motor cortex through the intrinsic modulation of sensory afference. However, whether other cognitive systems, like working memory, have the same influence upon motor cortex and/or act upon the same motor cortical substrates is unknown.

This study assessed the effect of short-term memory demands on the specific afferent intracortical motor circuits recruited by different monophasic current directions. SAI was elicited using either PA or AP TMS to preferentially recruit distinct sensory-motor cortical circuits (Ni et al., 2011) during memory set maintenance in the Sternberg short-term memory task (Sternberg, 1966). Psychophysical studies demonstrate strong interactions between attention and working memory in the processing of sensory stimuli (Lavie, 2010). Therefore, we hypothesized that increasing the set size to be maintained in working memory would, at least, influence sensory-motor afferent projections in the attentionsensitive AP circuit (Mirdamadi et al., 2017). In a second independent experiment, we directly investigated the effect of working memory load upon somatosensory processing. The parietal N20-P25 and frontal P20-N30 somatosensoryevoked potentials were elicited using median nerve stimulation during the working memory task and working memory loads as used to study SAI. The amplitude of the parietal N20-P25 is positively correlated with the magnitude of PA SAI (Bailey et al., 2016) while we have shown similar attention-related decreases in the P20-N30 and AP SAI (Mirdamadi et al., 2017). We hypothesized working memory effects upon PA and AP SAI would be mirrored in the N20-P25 and P20-N30 SEP components, respectively.

\section{2 | MATERIALS AND METHODS}

\section{1 | Participants}

Nineteen self-reported right-handed adults participated in the original TMS experiment assessing SAI during the maintenance of different digit spans (Experiment 1; 6 males, 13 females, $20 \pm 2$ years). An independent sample of ten selfreported right-handed adults participated in the subsequent somatosensory-evoked potential experiment (Experiment 2; 4 males, 6 females, $20 \pm 1$ years). All participants across both experiments provided written informed consent; the Institutional Review Board of the University of Michigan Medical School (IRBMED) approved the study protocol.

\section{2 | Working memory task}

For both experiments, the working memory task was a modified version of the Sternberg short-term memory task (Figure 1) (Sternberg, 1966). Sets of two- or six-digits were randomly presented for $2,000 \mathrm{~ms}$ on a computer screen $80 \mathrm{~cm}$ in front of the participant (Labview 2015, National Instruments, Austin, Texas, USA). After 2,000 ms the memory set disappeared. Following a 2,000 ms delay consisting of a blank, gray background, a single digit probe appeared. Participants indicated whether the probe was part of the previously presented memory set by pressing one of two response keys located beneath the left index or middle finger. The single digit probe remained visible until participants responded or 2,000 ms elapsed. The inter-trial interval was $2,000 \mathrm{~ms}$. The probability that the probe was included in the memory set was $50 \%$. Response time and accuracy were recorded. Participants completed 15 trials for each combination of TMS current direction and memory load. 


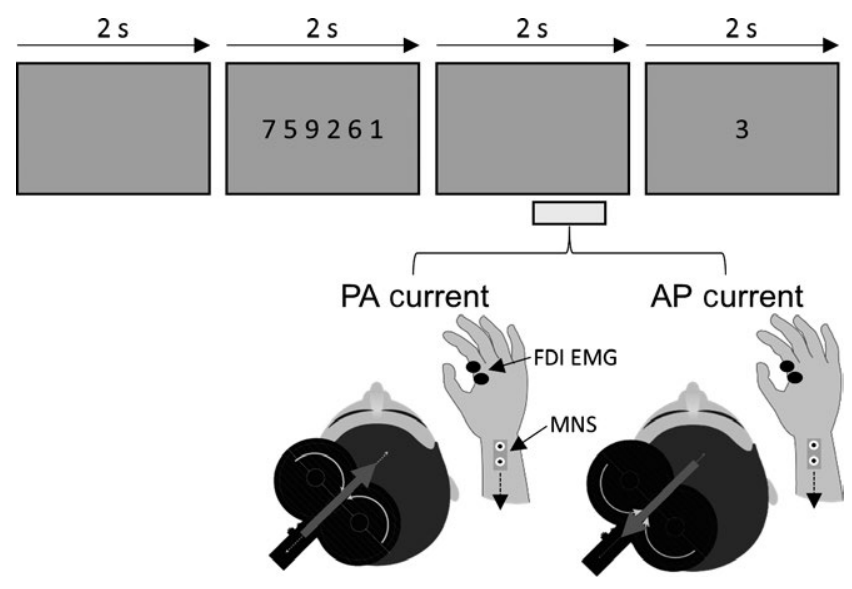

F I G U R E 1 Example time course of the Sternberg shortterm memory task and timing of SAI assessment. Memory set size consisted of either a two-digit or six-digit memory set to be encoded and maintained. SAI under different current directions was elicited 1,000-1,750 ms following memory set disappearance (light grey bar). The dark grey arrows indicate the induced current in the brain. The white curved arrows on the TMS coil indicate the direction of the TMS coil current. Note, the induced current in the brain flows in the opposite direction to the coil current. The TMS coil current direction was controlled by the stimulator's onboard software. FDI EMG - first dorsal interosseous electromyography electrode, MNS - median nerve stimulation

\section{3 | Transcranial Magnetic Stimulation (TMS)}

For Experiment 1, motor-evoked potentials (MEP) elicited by TMS during the 2,000 ms delay (i.e., memory maintenance) were recorded using LabChart 7 software in conjunction with a Dual BioAmp and PowerLab 8/30 acquisition system (AD Instruments, Colorado Springs, Colorado, USA). Participants sat with both arms resting on a pillow placed upon their lap. Surface electromyography electrodes $(\mathrm{Ag}-\mathrm{AgCl})$ were placed over the first dorsal interosseous muscle, using a tendon-belly montage. Surface electromyography recording was triggered using a $5 \mathrm{~V}$ TTL pulse with an epoch of -0.3 to $0.5 \mathrm{~s}$. During acquisition, data were amplified $(\times 1,000)$, digitized $(\times 40,000 \mathrm{~Hz})$ and filtered (band pass filtered 5-1,000 Hz, notch filter $-60 \mathrm{~Hz}$ ). Surface electromyography data were subsequently down-sampled to $5,000 \mathrm{~Hz}$ during offline analysis. The MEP was defined as the peak-to-peak amplitude of the maximal electromyography response between 20 and $50 \mathrm{~ms}$ post-TMS stimulation. Trials were excluded from subsequent analysis if the root mean square error of the electromyographic signal from the first dorsal interosseous muscle exceeded $15 \mu \mathrm{V}$ during the pre-TMS stimulus interval ( -50 to $0 \mathrm{~ms}$ ). Less than $1 \%$ of all trials were excluded because of excessive muscle activity during the pre-TMS stimulus interval.
TMS was delivered using a MagVenture MagPro X100 with option stimulator (MagVenture Inc., Atlanta, Georgia, USA) and a figure-8 coil (MC-B70). Two different current configurations were delivered. For PA stimulation, the coil was held $\sim 45^{\circ}$ to the midline and current direction specific as "reverse" using the stimulator's onboard software (Figure 1). For AP stimulation, the coil orientation was identical with that for PA stimulation but current direction was current was set to "normal" via the stimulator's onboard software (Figure 1).

The first dorsal interosseous motor cortical hotspot was defined as the scalp position that elicited the largest and most consistent response following PA stimulation. The location and trajectory of the coil on the scalp at the hotspot was recorded using the BrainSight ${ }^{\mathrm{TM}}$ stereotactic system (Rogue Research, Montreal, Québec, Canada). The same hotspot was used for AP stimulation (Sakai et al., 1997). Resting motor threshold was independently defined for the PA and APinduced currents as the percentage of stimulator output (to the nearest $1 \%$ ) that elicited an MEP of $\geq 50 \mu \mathrm{V}$ peak-to-peak on 10 out of 20 trials (Rossini et al., 2015). TMS intensity for SAI was set to the stimulator output that elicited an MEP of $\sim 1 \mathrm{mV}$ (in the absence of peripheral stimulation) for each current direction (Ni et al., 2011).

\section{4 | Short-Latency Afferent Inhibition (SAI)}

Short-Latency Afferent Inhibition consisted of a peripheral electrical stimulus paired with TMS. Electrical stimulation was delivered, using a DS7A constant current high voltage stimulator (Digitimer North America LLC, Fort Lauderdale, Florida, USA). Stimulation was applied over the median nerve at the right wrist (constant current square wave pulse, $0.2 \mathrm{~ms}$ duration, cathode proximal). Electrical stimulation intensity was set to the intensity to produce a slight thumb twitch (Abbruzzese, Marchese, Buccolieri, Gasparetto, \& Trompetto, 2001). The electrical stimulus intensity was $2.6 \pm 0.9$ times sensory threshold. Electrical stimulation preceded TMS stimulation by $21 \mathrm{~ms}$, an ISI known to produce the strongest inhibition for PA (Ni et al., 2011; Tokimura et al., 2000) and AP SAI (Ni et al., 2011).

\section{5 | Somatosensory-evoked Potentials}

For Experiment 2, somatosensory-evoked potentials were derived from electrical stimulation to the right median nerve during the memory maintenance period (i.e., after the disappearance of the memory set but before the appearance of the probe digit). Median nerve stimulation consisted of square wave pulses (0.5 ms duration) delivered through surface electrodes fixed to the wrist (DS7A constant current stimulator, Digitimer North America LLC, Fort Lauderdale, Florida, 
USA). Median nerve stimulus intensity was set to motor threshold and the $\mathrm{M}$-wave was monitored via surface electromyography at the thenar eminence $(\times 2,000$ amplification, $1-200 \mathrm{~Hz}$ bandpass filtered, digitized at $1,200 \mathrm{~Hz}$, g.tec g.USBAmp, g.tec Neurotechnology, Rensselaer, New York, USA).

Electroencephalographic data were recorded from $\mathrm{Fz}$, $\mathrm{Cz}, \mathrm{Pz}, \mathrm{Oz}, \mathrm{C} 3, \mathrm{C} 4, \mathrm{Cp} 3, \mathrm{Cp} 4$, and A1 (g.Sahara active dry electrodes, g.UBSamp, g.tec medical engineering GMBH, Schiedlberg, Austria). Electrodes were positioned consistent with the international 10-20 system for electrode placement and referenced to the right mastoid ( $<5 \mathrm{k} \Omega$ impedance, $\times 20,000$ amplification, $1-200 \mathrm{~Hz}$ bandpass filtered, $1,200 \mathrm{~Hz}$ digitization). Somatosensory-evoked potentials were extracted offline, using the EEGLab toolbox (Institute for Neural Computation, University of California-San Diego, San Diego, California, USA) for MATLAB v2017a (The MathWorks, Natick, Massachusetts, USA) environment. First the electroencephalographic data were re-referenced to the left mastoid (A1). Data were visually inspected to remove trials and channels that were contaminated by noise. In two subjects, Oz was removed from subsequent analysis. An independent component analysis was performed to remove eye-related artifacts. To reduce the effect of the stimulation artefact upon the subsequent analyses an epoch ranging from -5 to $10 \mathrm{~ms}$ around the stimulation artefact was removed from each trial and interpolated using spline interpolation. The continuous data were then filtered (Butterworth bandpass filter, $5-100 \mathrm{~Hz}$, $24 \mathrm{~dB} /$ Octave). Approximately, 180 epochs per condition per subject were extracted ( -500 to $500 \mathrm{~ms}$ relative to median nerve stimulus onset), baseline corrected ( -50 to $0 \mathrm{~ms}$ ) and averaged. Peak-to-peak amplitudes for the frontal P20-N30 and parietal N20-P25 were extracted from Fz and Cp3, respectively.

\subsection{Experimental design and procedure}

Experiment 1 consisted of a single session. SAI was elicited during the 2-s maintenance period between the two- or sixdigit span presentation and presentation of the probe. The timing of the stimulus during the memory maintenance period was variable, determined by a rectangular distribution with a mean of $1,375 \mathrm{~ms}$ and a range of $1,000-1,750 \mathrm{~ms}$. During each trial, a single unconditioned or conditioned stimulus was delivered. Fifteen conditioned and fifteen unconditioned trials were completed for each digit span length and monophasic current direction resulting in a total of 120 trials. The order of conditioned/unconditioned epochs, current direction and span length was counterbalanced across participants.

Experiment 2 also consisted of a single session. Somatosensory-evoked potentials were elicited during the 2-s maintenance period between the two- or six-digit span presentation and presentation of the probe. During each trial, four median nerve stimuli were delivered during the maintenance period, On each trial, the timing of the four median nerve stimuli was fixed at 400, 900, 1,400 and 1,900 ms following the disappearance of the memory set. Participants completed ninety-trials of the Sternberg memory task in total. Forty-five trials used a set size of two. The remaining 45 trials used a set size of six. The order of the working memory load trials was randomized. It should also be noted that the Sternberg working memory trials were completed as part of a larger study that also included elicitation of somatosensoryevoked potentials during performance of a visual detection task. The order of attention versus working memory was counterbalanced across participants.

\section{7 | Data analysis}

Statistical analyses were performed using IBM SPSS Statistics for Windows (v23, IBM Corp. Armonk, New York, USA). For Experiment 1, behavioral performance was assessed using repeated measures ANOVA with the factors Set Size (Two, Six) and Current Direction (PA, AP).

Altered sensory-motor cortical physiology across working memory set size was assessed using a Current Direction (PA, AP) by Set Size (Two, Six) repeated measures ANOVA. SAI was the dependent variable. SAI was derived as a percentage of unconditioned MEP amplitude. Lower values for SAI indicate higher levels of MEP suppression by the somatosensory afferent volley.

For Experiment 2, the peak-to-peak amplitudes of the parietal N20-P25 and frontal P20-N30 somatosensory-evoked potentials were compared across the two- and six-digit set sizes using Set Size (Two, Six) by Component (parietal N20-P25, frontal P20-N30) repeated measures ANOVA. The significant interaction was decomposed using separate contrasts comparing low versus high load for each somatosensory-evoked potential.

\section{3 | RESULTS}

\section{1 | Experiment 1 - Monophasic TMS during maintenance of varying set size}

Mean accuracy in the visual detection task was higher for the two- compared to six-digit memory set size [Main Effect $_{\text {Set Size: }} F_{1,18}=26.49, p=0.00008$; Two $=96 \pm 1 \%$, Six $=89 \pm 2 \%$, mean \pm standard error]. Accuracy was consistent across current direction as neither the interaction $\left[F_{1,18}=0.03, p=0.88\right]$ nor the main effect of Current Direction $\left[F_{1,18}=0.35, p=0.56\right]$ were significant.

Figure 2a shows the group averaged traces $(n=19)$ of conditioned and unconditioned MEPs for each current direction. Resting motor threshold for monophasic PA stimulation and AP stimulation was $49 \pm 6$ (mean \pm standard deviation) and $66 \pm 9 \%$ of stimulator output, respectively. The stimulation intensity to elicit an MEP of $1 \mathrm{mV}$ using monophasic PA and AP stimulation was $58 \pm 8 \%$ and $78 \pm 10 \%$ of stimulator output, respectively. Consistent with past work, the latency of MEPs was significantly longer 

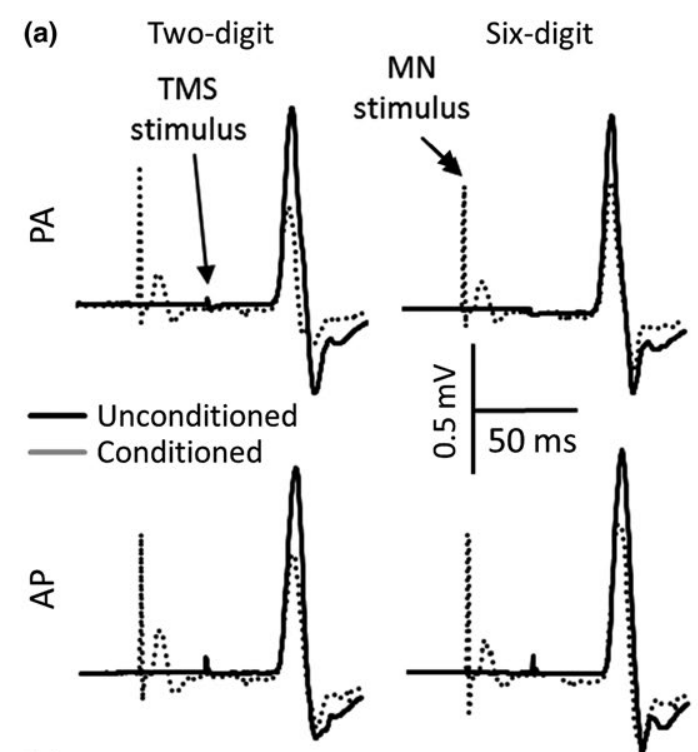

(b)

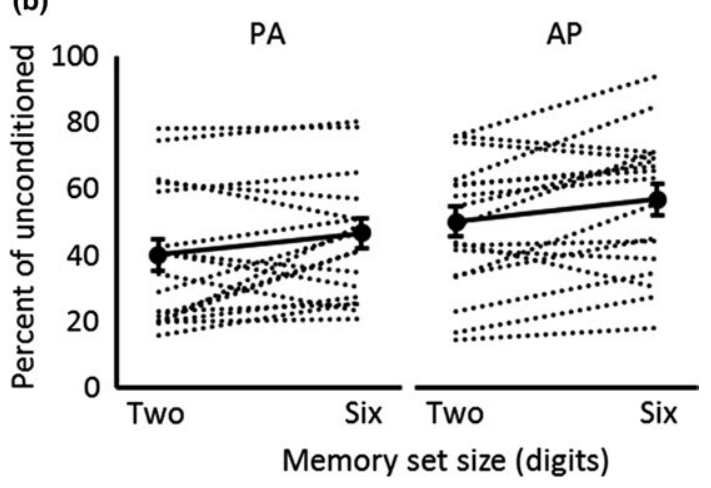

F I G URE 2 (a) Unconditioned (solid) and conditioned (dashed) group averaged motor-evoked potentials elicited by PA and AP stimulation during the two- (left panel) and six-digit (right panel) conditions. (b) SAI elicited during maintenance of the two- and six-digit memory sets. Amplitudes are expressed as a percentage of the unconditioned motor-evoked potential amplitude. Higher values represent lower levels of SAI. The group average across the two- and six-digit set sizes for each current direction (solid line) is overlaid on individual data (dashed lines). Error bars represent standard error of the mean

for AP compared to PA stimulation [Main Effect $_{\text {Current }}$ Direction: $F_{1,18}=31.11, p=0.00003, \mathrm{PA}=22.5 \pm 1.1 \mathrm{~ms}$, $\mathrm{AP}=24.1 \pm 1.1 \mathrm{~ms}$, mean \pm standard error]. The longer latencies for AP current were consistent across digit set size given the absence of a significant main effect of Set Size $\left[F_{1,18}=2.12, p=0.16\right]$ or Set Size by Current Direction interaction $\left[F_{1,18}=0.12, p=0.73\right]$.

Short-latency Afferent Inhibition was significantly reduced during memory maintenance of the six-digit compared to two-digit set [Main Effect ${ }_{\text {Set Size }}: F_{1,18}=16.55, p=0.001$ ] (Figure $2 \mathrm{~b}$ ). The effect of memory set size was consistent across current direction as neither the main effect of Current Direction $\left[F_{1,18}=2.61, p=0.12\right]$ nor the Current Direction by Set Size interaction $\left[F_{1,18}<0.0001, p=0.99\right]$ reached significance.

\section{2 | Experiment 2 - Somatosensory gating under varying cognitive load}

Figure $3 \mathrm{a}$ and $\mathrm{b}$ show the group averaged SEPs $(n=10)$ from $\mathrm{Fz}$ and $\mathrm{Cp} 3$. The effect of memory set size varied depending upon the somatosensory sensory-evoked potential [Interaction ${ }_{\text {Set SizexComponent }} ; F_{1,9}=12.99, p=0.006$ ]. Decomposition revealed that the amplitude of the parietal N20-P25 was significantly reduced during the maintenance of the six-digit compared to two-digit memory set $(p=0.018)$ (Figure 3c). In contrast, the amplitude of the frontal P20-N30
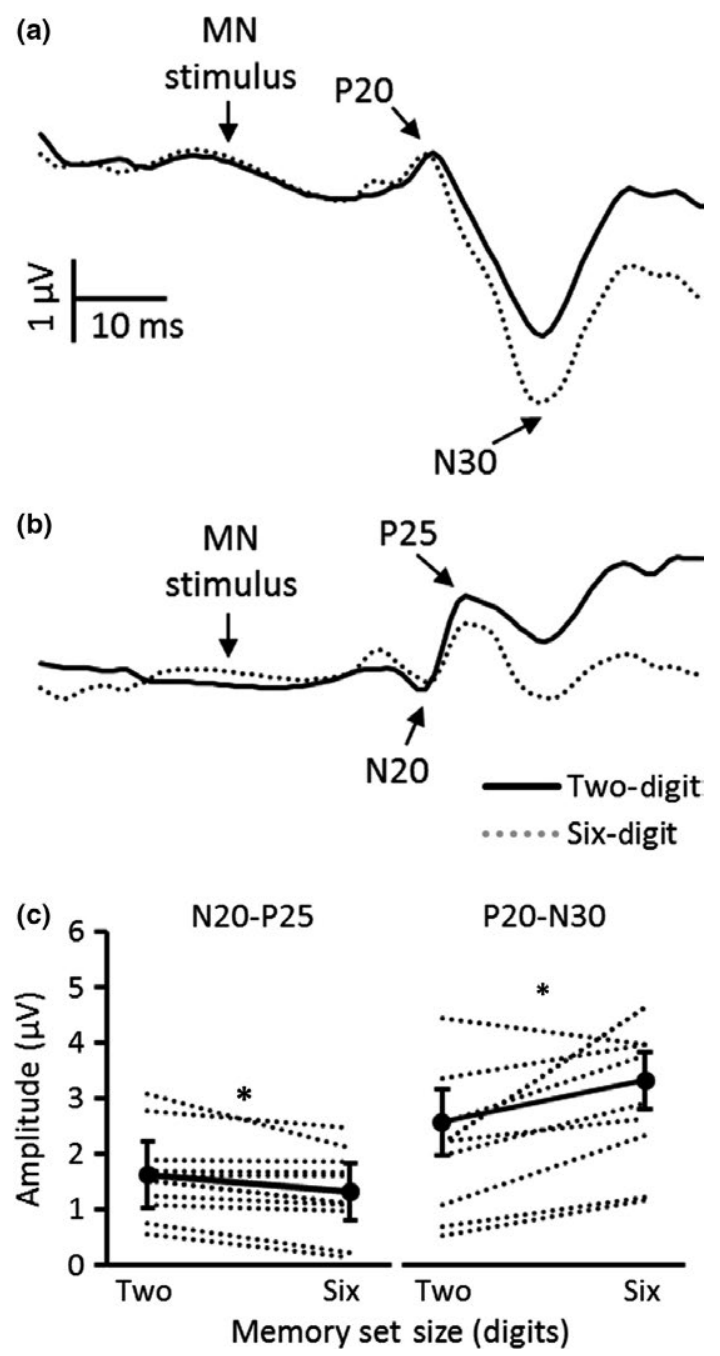

F IGURE 3 (a) Grand average SEP waveform recorded from $\mathrm{Fz}$ elicited during maintenance of the two- (solid) and six-digit (dashed) memory sets. (b) Grand average SEP waveform recorded from Cp3 elicited during maintenance of the two- (black) and six-digit (grey) memory sets. (c) Peak-to-peak amplitudes of the parietal N20-P25 and frontal P20-N30 during maintenance of the two- and six-digit memory sets. The group average across the two- and six-digit conditions for each SEP component (solid line) is overlaid on individual data (dashed lines). Error bars represent standard error of the mean. *denotes significant contrast $(p<0.05)$ 
SEP amplitude was increased during the maintenance of the six-digit compared to two-digit memory set $(p=0.014)$ (Figure 3c).

\section{4 | DISCUSSION}

This study used SAI to investigate the effects of cognitive load upon afferent intracortical circuits. The novel finding is that higher verbal working memory loads associated with the maintenance of a six- versus two-digit set reduced SAI regardless of whether SAI was evoked using PA or AP stimulating current. The reduction in SAI was mirrored by a reduction in the parietal N20-P25 somatosensory-evoked potential in a separate sample.

The general reduction in PA and AP SAI with increasing memory set size is consistent with increased suppression of the task-irrelevant somatosensory afferent projections to motor cortex during the maintenance period of the verbal working memory task. The suppression of task-irrelevant somatosensory afference with increasing working memory demands of a concurrent visual task is consistent with a report of increased suppression of early auditory event-related potentials with increasing working memory demands on a concurrent visual task (Simon, Tusch, Holcomb, \& Daffner, 2016). It should be noted that while the reduction in SAI across current direction was statistically significant at the group level there was variability across participants within each current direction. In particular, a small subset of individuals demonstrated increased SAI with increasing set size. Two possible explanations for the variability may relate to working memory capacity and/or strategy. A limitation of the current study is that we did not assess working memory capacity or control for strategy during task performance. Future work is needed to better quantify the sources of individual variability. Despite the small cohort demonstrating SAI variance opposite of the group, the mirrored reduction in N20-P25 SEP component amplitude when memory set size is increased from two to six digits is consistent with the overall trend of increased suppression of task-irrelevant somatosensory afference during the memory maintenance period. Although SEPs were evoked using consistent median nerve stimulation with the SAI procedure and during the same memory maintenance period of the working memory task, a limitation to this study is that SAI and SEPs were recorded across two separate samples. Therefore, we cannot directly correlate the extent of SAI reduction with the reduction in the N20-P25 SEP component when moving from the two- to six-digit set size. However, given the strong correlation between SAI and the N20-P25 amplitude (Bailey et al., 2016), altered sensory-motor processing dependent upon the crossmodal allocation of resources likely provides a potential substrate by which declarative cognitive strategies may shape procedural motor control in healthy and clinical populations. In particular, SAI may provide a marker of cholinergic influence (Ziemann et al., 2014) tied to different strategies or foci of attention that can interact with other facilitatory (Cash, Isayama, Gunraj, Ni, \& Chen, 2015) and inhibitory (Alle, Heidegger, Krivanekova, \& Ziemann, 2009; Udupa, $\mathrm{Ni}$, Gunraj, \& Chen, 2013) intracortical motor circuits critical to skilled performance and learning.

The common reduction in PA and AP SAI with increasing memory set size suggests a functionally correlated influence across distinct anatomical circuits converging on the corticospinal neuron. The evoked muscular response induced by magnetic stimulation of the brain is driven by a series of transynaptic projections on to the corticospinal neuron termed I-waves (Di Lazzaro et al., 2012b). These transynaptic projections are thought to reflect independent intracortical circuits with distinct input pathways to the corticospinal neuron (Di Lazzaro et al., 2001; Ni et al., 2011). PA stimulating current predominantly elicits early I1- and I2-waves whereas AP-stimulating current favors recruitment of I3- and I4-waves (Di Lazzaro et al., 2001). Functionally, PA- and AP-sensitive circuits have been linked to model-free and model-based motor learning (Hamada et al., 2014) while we have demonstrated a differential selective sensitivity of AP SAI to visual attention load (Mirdamadi et al., 2017). One possibile explanation for the common reduction across PA and AP SAI is that increased working memory load targeted the same corticospinal input (i.e., I-wave generator) across stimulating current direction. Indeed PA stimulating current predominantly evokes early I-waves, yet epidural recordings during magnetic stimulation show that PA current at high intensities can evoke I3- and I4-waves (Di Lazzaro et al., 2001; Tokimura et al., 2000). However, such studies set stimulation intensity based upon the criterion of much larger MEPs $(\geq 2 \mathrm{mV})$ than employed here $(\sim 1 \mathrm{mV})$. Therefore, it is likely that stimulation intensity in those studies was much higher than employed here. Previous work has dissociated PA and AP SAI using the same $1 \mathrm{mV}$ test stimulus as employed in this study (Ni et al., 2011) making it less likely that the common reduction across PA and AP SAI is the result of a common late I-wave generator.

A second explanation for the common reduction in SAI across PA and AP circuits is the influence of a mechanism downstream from the corticospinal input of both the early and late I-wave generators. A recent neuronal model of SAI (Turco et al., 2018) proposes that sensory afference drives corticospinal activity through a perisomatic inhibitory projection as well as the modulation of distal dendritic excitatory input from the I-wave generators. The perisomatic inhibitory input is proposed to originate from $\gamma$-amino butyric acid (GABA) basket cells located in layer IV of the motor cortex. These basket cells are hypothesized to receive excitatory input from somatosensory pyramidal neurons as well as the 
thalamus itself and are sensitive to cholinergic activity (Szabo, Holderith, Gulyas, Freund, \& Hajos, 2010). Decreased excitation of the inhibitory basket cells by upstream gating of their somatosensory afferent input could commonly decrease PA and AP SAI by depolarizing the perisomatic region of the corticospinal neuron downstream from both the early and late I-wave inputs (Turco et al., 2018). Under this model, sensory afference may still influence corticospinal excitability across multiple distinct pathways despite the common decrease across stimulating current observed here to maintain a level of functional flexibility. In particular, the perisomatic effect may provide a mechanism to facilitate desired or suppress undesired/conflicting actions while other sensory-motor circuits may provide the ability to modulate ongoing actions. Such a dissociation would be consistent with reports that SAI is reduced during the preparatory phase immediately preceding finger movement (Asmussen et al., 2013; Voller et al., 2006) and serves to inhibit surrounding digit representations not involved in the planned movement (Asmussen et al., 2014; Dubbioso et al., 2017; Voller et al., 2006). In turn, the selective sensitivity of AP SAI to perceptual factors (Mirdamadi et al., 2017) highlights a parallel, functionally distinct cholinergic mechanism to provide a flexibility and complexity to support different behaviors governed by motor cortex (Hamada et al., 2014).

The mediation of working memory effects by an indiscriminate mechanism is supported by reduced parietal N20P25 but increased P20-N30 SEP component amplitude when the memory set is increased from two to six digits. We previously demonstrated that reduced AP SAI with increased visual attention load was mirrored by a reduction in the frontal P20-N30 but not parietal N20-P25 SEP component (Mirdamadi et al., 2017). The frontal P20-N30 generator has been localized to the supplementary motor area and precentral gyrus (Desmedt \& Cheron, 1981) consistent with the hypothesized origins of later I-waves recruited by AP TMS (Di Lazzaro et al., 2012b; Volz, Hamada, Rothwell, \& Grefkes, 2014). Therefore, the increase in P20-N30 amplitude may reflect a distinct perceptual mechanism where gating of sensory afference is released under high working memory but whose influence upon the corticospinal neurons of motor cortex can be over-ridden by a functionally independent perisomatic mechanism tied to response selection.

The common effect of working memory and attention upon AP SAI across our current and past work also raises interesting possibilities tied to TMS variability in healthy and clinical populations. In primary motor cortex, the propensity of TMS stimuli to recruit later I-waves during repetitive stimulation protocols is a strong predictor of the magnitude and direction of the subsequent after-effect (Hamada, Murase, Hasan, Balaratnam, \& Rothwell, 2013).The consistent reduction in AP SAI across attention and working memory highlights potentially important mediators of corticospinal excitation by TMS (Di Lazzaro et al., 2012a). While past work has demonstrated the sensitivity of traditional repetitive TMS (Conte et al., 2007), intermittent theta burst (Kamke et al., 2012) and paired associative stimulation (Kamke et al., 2012, 2014; Stefan et al., 2004) plasticity inducing protocols to spatial and cross-modal attention, this study suggests similar work investigating the influence of working memory is needed.

This study provides converging evidence that direct sensory-motor projections are influenced by the allocation of cognitive resources. The common sensitive of AP circuits to both working memory and attention but the selective sensitivity of PA circuits to working memory may reflect distinct pathways by which declarative strategies may influence procedural processing in motor control and learning.

\section{ACKNOWLEDGMENTS}

The present work was partially supported by a KL2 Scholar award to SKM from the Claude D. Pepper Older Americans Independence Center at the University of Michigan (P30AG024824). LYS was partially supported by the KL2 scholar award to SKM. The authors thank Adam Feingold and Dalia Khammash for their help during data collection.

\section{CONFLICT OF INTEREST STATEMENT}

None of the authors have potential conflicts of interest to be disclosed.

\section{DATA ACCESSIBILITY STATEMENT}

Data and analysis scripts are available from the corresponding author, SKM, upon request.

\section{AUTHOR CONTRIBUTIONS}

All authors had full access to all the data in the study and take responsibility for the integrity of the data and the accuracy of the data analysis. Study concept and design: LYS, SKM. Acquisition of data: LYS. Analysis and interpretation of data: LYS, SKM. Drafting of the manuscript: LYS, SKM. Obtained funding: SKM.

\section{ORCID}

Sean K. Meehan (D) http://orcid.org/0000-0002-9848-8803

\section{REFERENCES}

Abbruzzese, G., Marchese, R., Buccolieri, A., Gasparetto, B., \& Trompetto, C. (2001). Abnormalities of sensorimotor integration 
in focal dystonia: A transcranial magnetic stimulation study. Brain, 124, 537-545. https://doi.org/10.1093/brain/124.3.537

Alle, H., Heidegger, T., Krivanekova, L., \& Ziemann, U. (2009). Interactions between short-interval intracortical inhibition and short-latency afferent inhibition in human motor cortex. The Journal of Physiology-London, 587, 5163-5176. https://doi.org/10.1113/ jphysiol.2009.179820

Asmussen, M. J., Jacobs, M. F., Lee, K. G., Zapallow, C. M., \& Nelson, A. J. (2013). Short-latency afferent inhibition modulation during finger movement. PLoS ONE, 8, e60496. https://doi.org/10.1371/ journal.pone.0060496

Asmussen, M. J., Zapallow, C. M., Jacobs, M. F., Lee, K. G. H., Tsang, P., \& Nelson, A. J. (2014). Modulation of short-latency afferent inhibition depends on digit and task-relevance. PLOS ONE, 9, 12.

Bailey, A. Z., Asmussen, M. J., \& Nelson, A. J. (2016). Short-latency afferent inhibition determined by the sensory afferent volley. Journal of Neurophysiology, 116, 637-644. https://doi.org/10.1152/ jn.00276.2016

Cash, R. F., Isayama, R., Gunraj, C. A., Ni, Z., \& Chen, R. (2015). The influence of sensory afferent input on local motor cortical excitatory circuitry in humans. Journal of Physiology, 593, 1667-1684. https:// doi.org/10.1113/jphysiol.2014.286245

Conte, A., Gilio, F., Iezzi, E., Frasca, V., Inghilleri, M., \& Berardelli, A. (2007). Attention influences the excitability of cortical motor areas in healthy humans. Experimental Brain Research, 182, 109-117. https://doi.org/10.1007/s00221-007-0975-3

Desmedt, J. E., \& Cheron, G. (1981). Non-cephalic reference recording of early somatosensory potentials to finger stimulation in adult or aging normal man: Differentiation of widespread N18 and contralateral N20 from the prerolandic P22 and N30 components. Electroencephalography and Clinical Neurophysiology, 52, 553-570. https://doi. org/10.1016/0013-4694(81)91430-9

Di Lazzaro, V., Oliviero, A., Saturno, E., Pilato, F., Insola, A., Mazzone, P., ... Rothwell, J. C. (2001). The effect on corticospinal volleys of reversing the direction of current induced in the motor cortex by transcranial magnetic stimulation. Experimental Brain Research, 138, 268-273. https://doi.org/10.1007/s002210100722

Di Lazzaro, V., Profice, P., Pilato, F., Capone, F., Ranieri, F., Florio, L., ... Dileone, M. (2012a). The level of cortical afferent inhibition in acute stroke correlates with long-term functional recovery in humans. Stroke, 43, 250-252. https://doi.org/10.1161/ STROKEAHA.111.631085

Di Lazzaro, V., Profice, P., Ranieri, F., Capone, F., Dileone, M., Oliviero, A., \& Pilato, F. (2012b). I-wave origin and modulation. Brain Stimulation, 5, 512-525. https://doi.org/10.1016/j.brs.2011.07.008

Dubbioso, R., Raffin, E., Karabanov, A., Thielscher, A., \& Siebner, H. R. (2017). Centre-surround organization of fast sensorimotor integration in human motor hand area. NeuroImage, 158, 37-47. https:// doi.org/10.1016/j.neuroimage.2017.06.063

Fischer, M., \& Orth, M. (2011). Short-latency sensory afferent inhibition: Conditioning stimulus intensity, recording site, and effects of $1 \mathrm{~Hz}$ repetitive TMS. Brain Stimulation, 4, 202-209. https://doi. org/10.1016/j.brs.2010.10.005

Hamada, M., Galea, J. M., Di Lazzaro, V., Mazzone, P., Ziemann, U., \& Rothwell, J. C. (2014). Two distinct interneuron circuits in human motor cortex are linked to different subsets of physiological and behavioral plasticity. Journal of Neuroscience, 34, 12837-12849. https://doi.org/10.1523/JNEUROSCI.1960-14.2014
Hamada, M., Murase, N., Hasan, A., Balaratnam, M., \& Rothwell, J. C. (2013). The role of interneuron networks in driving human motor cortical plasticity. Cerebral Cortex, 23, 1593-1605. https://doi. org/10.1093/cercor/bhs147

Kamke, M. R., Hall, M. G., Lye, H. F., Sale, M. V., Fenlon, L. R., Carroll, T. J., ... Mattingley, J. B. (2012). Visual attentional load influences plasticity in the human motor cortex. Journal of Neuroscience, 32, 7001-7008. https://doi.org/10.1523/JNEUROSCI.1028-12.2012

Kamke, M. R., Ryan, A. E., Sale, M. V., Campbell, M. E., Riek, S., Carroll, T. J., \& Mattingley, J. B. (2014). Visual spatial attention has opposite effects on bidirectional plasticity in the human motor cortex. Journal of Neuroscience, 34, 1475-1480. https://doi. org/10.1523/JNEUROSCI.1595-13.2014

Lavie, N. (2010). Attention, distraction, and cognitive control under load. Current Directions in Psychological, 19, 143-148. https://doi. org/10.1177/0963721410370295

Meehan, S. K., Legon, W., \& Staines, W. R. (2009). Spatiotemporal properties modulate intermodal influences on early somatosensory processing during sensory-guided movement. Clinical Neurophysiology, 120, 1371-1380. https://doi.org/10.1016/j. clinph.2009.05.005

Mirdamadi, J. L., Suzuki, L. Y., \& Meehan, S. K. (2017). Attention modulates specific motor cortical circuits recruited by transcranial magnetic stimulation. Neuroscience, 359, 151-158. https://doi. org/10.1016/j.neuroscience.2017.07.028

Ni, Z., Charab, S., Gunraj, C., Nelson, A. J., Udupa, K., Yeh, I. J., \& Chen, R. (2011). Transcranial magnetic stimulation in different current directions activates separate cortical circuits. Journal of Neurophysiology, 105, 749-756. https://doi.org/10.1152/ jn.00640.2010

Rossini, P. M., Burke, D., Chen, R., Cohen, L. G., Daskalakis, Z., Di Iorio, R., ... Ziemann, U. (2015). Noninvasive electrical and magnetic stimulation of the brain, spinal cord, roots and peripheral nerves: Basic principles and procedures for routine clinical and research application. An updated report from an I.F.C.N. Committee. Clinical Neurophysiology, 126, 1017-1107.

Ruge, D., Muggleton, N., Hoad, D., Caronni, A., \& Rothwell, J. C. (2014). An unavoidable modulation? Sensory attention and human primary motor cortex excitability. European Journal of Neuroscience, 40, 2850-5858. https://doi.org/10.1111/ejn.12651

Sakai, K., Ugawa, Y., Terao, Y., Hanajima, R., Furubayashi, T., \& Kanazawa, I. (1997). Preferential activation of different I waves by transcranial magnetic stimulation with a figure-of-eight-shaped coil. Experimental Brain Research, 113, 24-32. https://doi.org/10.1007/ BF02454139

Simon, S. S., Tusch, E. S., Holcomb, P. J., \& Daffner, K. R. (2016), Increasing working memory load reduces processing of crossmodal task-irrelevant stimuli even after controlling for task difficulty and executive capacity. Frontiers in Human Neuroscience, $10,380$.

Stefan, K., Wycislo, M., \& Classen, J. (2004). Modulation of associative human motor cortical plasticity by attention. Journal of Neurophysiology, 92, 66-72. https://doi.org/10.1152/jn.00383.2003

Sternberg, S. (1966). High-speed scanning in human memory. Science, 153, 652-654. https://doi.org/10.1126/science.153.3736.652

Szabo, G. G., Holderith, N., Gulyas, A. I., Freund, T. F., \& Hajos, N. (2010). Distinct synaptic properties of perisomatic inhibitory cell types and their different modulation by cholinergic receptor activation in the $\mathrm{CA} 3$ region of the mouse hippocampus. European 
Journal of Neuroscience, 31, 2234-2246. https://doi.org/10.1111/ (ISSN)1460-9568

Tokimura, H., Di Lazzaro, V., Tokimura, Y., Oliviero, A., Profice, P., Insola, A., ... Rothwell, J. C. (2000). Short latency inhibition of human hand motor cortex by somatosensory input from the hand. Journal of Physiology, 523, 503-513. https://doi. org/10.1111/j.1469-7793.2000.t01-1-00503.x

Turco, C. V., El-Sayes, J., Savoie, M. J., Fassett, H. J., Locke, M. B., \& Nelson, A. J. (2018). Short- and long-latency afferent inhibition; uses, mechanisms and influencing factors. Brain Stimulation, 11, 59-74. https://doi.org/10.1016/j.brs.2017.09.009

Udupa, K., Ni, Z., Gunraj, C., \& Chen, R. (2013). Effects of short latency afferent inhibition on short interval intracortical inhibition. Journal of Neurophysiology, 111, 1350-1361.

Voller, B., St Clair Gibson, A., Dambrosia, J., Pirio Richardson, S., Lomarev, M., Dang, N., \& Hallett, M. (2006). Short-latency afferent inhibition during selective finger movement. Experimental Brain Research, 169,226-231.https://doi.org/10.1007/s00221-005-0140-9

Volz, L. J., Hamada, M., Rothwell, J. C., \& Grefkes, C. (2014). What makes the muscle twitch: Motor system connectivity and TMSinduced activity. Cerebral Cortex, 25, 2346-2453.

Ziemann, U., Reis, J., Schwenkreis, P., Rosanova, M., Strafella, A., Badawy, R., \& Muller-Dahlhaus, F. (2014). TMS and drugs revisited 2014. Clinical Neurophysiology, 126, 1847-1868.

How to cite this article: Suzuki LY, Meehan SK. Verbal working memory modulates afferent circuits in motor cortex. Eur J Neurosci. 2018;48:3117-3125. https://doi.org/10.1111/ejn.14154 